\title{
Um Ambiente de Autoria de Jogos Sérios pelo Usuário Final Aplicados a Educação
}

\author{
Alana Oliveira $^{1}$, Carlos de Salles Soares Neto ${ }^{1}$, Mário Meireles Teixeira ${ }^{1}$ \\ ${ }^{1}$ Universidade Federal do Maranhão \\ Programa de Pós-Graduação em Ciência da Computação \\ Laboratory of Advanced Web Systems - LAWS \\ Av. dos Portugueses, 1966 - Baganga - CEP 65080-805 - São Luís - MA \\ alana@nca.ufma.br, \{csalles, mario\}@deinf.ufma.br
}

\begin{abstract}
In an educational context, we are increasingly looking for tools that can assist the learning process. The use of games is usually believed to be an efficient means to try to gather students' attention, who are often distracted by an increasingly connected and interactive world. The challenge we intend to tackle in this project is to provide educators with tools to create their own games in a simple and straightforward manner, since they do not usually have computer programming skills. This paper proposes an authoring environment for serious games with educational purposes, which can be used by teachers and content managers to develop games. As proof of concept, games were produced for different domains by applying the tool. The usability of this tool was assessed through Norm ISO 9241-110, which was applied to different user groups, reaching very positive results .
\end{abstract}

Keywords: Serious Games, Educational Games, Authoring environment, EndUser Development.

Resumo. No contexto educacional, cada vez mais se buscam ferramentas que possam contribuir para facilitar o aprendizado. $O$ uso de jogos tem sido visto com bons olhos para tentar resgatar a atenção do aluno, muitas vezes distraído por um mundo cada vez mais conectado e interativo. Desta forma, o desafio que se tenciona enfrentar neste trabalho é dotar os educadores de ferramentas necessárias para produzir seus próprios jogos de maneira simples e direta, uma vez que eles não possuem, na maioria das vezes, habilidades de programação de computadores. O presente artigo propõe um ambiente de autoria de jogos sérios com propósitos educacionais, que pode ser utilizado por professores e gestores de conteúdo para o desenvolvimento de jogos. Como prova de conceito, foram produzidos jogos para diferentes domínios com a aplicação da ferramenta. A usabilidade desta foi avaliada através da Norma ISO 9241-110 com diferentes grupos de usuários, alcançando-se resultados bastante positivos.

Palavras-chave: Jogos Sérios, Jogos Educacionais, Ambiente de autoria, Desenvolvimento pelo usuário final.

\section{Introdução}

O uso de ferramentas lúdicas como os jogos tem se tornado importante no contexto educacional, uma vez que os objetos de aprendizagem utilizados muitas vezes têm uma maneira 
pouco interessante ou pouco integrada à realidade social e regional, o que dificulta o processo de ensino aprendizagem [Antunes and Sabóia-Morais ]. Nesse sentido, o docente pode ser o agente de transformação e inovação do ensino, trazendo para sala de aula metodologias diferenciadas que permitam a construção do conhecimento pelos aprendizes. Segundo [de Lara 2004], os jogos vêm ganhando espaço na sala de aula com a intenção dos professores de tornar o ensino algo mais cativante. Cada jogo carrega em si um aprendizado intrínseco, sem o qual o jogador não seria capaz de avançar nas fases do jogo e chegar ao prêmio final. De forma análoga, assim deveria ser o processo educativo: cada aluno buscando conhecimento como um fim em si mesmo, de forma lúdica e em permanente motivação.

Existe uma categoria de jogos, conhecida como serious games, que utiliza a conhecida abordagem lúdica, ao mesmo tempo em que oferece atividades que favorecem a absorção de conceitos e habilidades cognitivas como raciocínio, abstração, linguagem, memória, atenção, criatividade, capacidade de resolução de problemas, entre outras. Desse modo, o termo jogos sérios (serious games) passou a ser utilizado para identificar uma categoria de jogos com propósito específico, ou seja, que extrapolam a ideia de entretenimento e oferecem outros tipos de experiências, como aquelas voltadas ao aprendizado e ao treinamento [Machado et al. 2009].

Pesquisas recentes realizadas em julho de 2013 [ElearningBrasil 2013] comprovam que $92 \%$ dos participantes consideram que a aplicação de jogos educativos ao eLearning pode incrementar o desempenho e a capacidade de demonstrar o quanto o aluno aprendeu, podendo este melhorar principalmente na fixação do conteúdo (40\%), na prática do que foi aprendido (27\%), na motivação para o estudo (13\%) e ainda que jogos podem ser usados para avaliar (10\%) e descontrair o aluno. A pesquisa também conclui que a grande maioria (92\%) dos entrevistados acredita que jogos podem ser empregados para informar e praticar habilidades, conferindo destreza e competência e que os jogos educativos contribuem para transformar a avaliação tradicional, de forma que os alunos não mais se sintam pressionados em fazê-la e sim empolgados em aprender algo novo.

Este trabalho tem como objetivo propor um ambiente de autoria de jogos sérios pelo usuário final, no caso o professor, visando facilitar a criação de jogos com fins educacionais. Como principais contribuições, destacam-se: modelagem conceitual e prototipação do ambiente de autoria de jogos sérios; identificação, modelagem e implementação de templates de jogos; avaliação de usabilidade da ferramenta; desenvolvimento de jogos como estudo de caso.

Em [Battistella and von Wangenheim 2011] diversas ferramentas foram avaliadas empiricamente, com as quais nossa ferramenta de autoria, denominada GReAT (Game Ready Authoring Tool) foi comparada. Esta assemelha-se às ferramentas $e X e$ Learning e Xerte por ser totalmente baseada em tecnologias Web, o que a torna imediatamente utilizável, sem nenhuma necessidade de configuração do ambiente por parte do usuário. A GReAT também não exige conhecimento de programação e permite a criação de objetos de aprendizagem através de um conjunto de telas, não sequenciais, que dirigem as ações do usuário. 


\section{Grupo de Foco}

A metodologia utilizada para coleta e validação dos requisitos do ambiente de autoria foi o grupo de foco [Nielsen 1997]. Esta técnica faz parte do processo de análise qualitativa e consiste em uma reunião de um grupo de pessoas conduzida por um mediador com o objetivo de promover discussão acerca de um tema, permitindo coletar múltiplos pontos de vista em um curto período de tempo. Um grupo de foco pode ser usado para gerar ideias, identificar conflitos relacionados a terminologia e identificar expectativas de diferentes grupos de pessoas [Barbosa and da Silva 2010].

Neste trabalho, o grupo de foco foi formado por 8 participantes, um mediador principal e um mediador auxiliar e as sessões foram gravadas em áudio e vídeo. O levantamento do perfil do grupo, visando principalmente avaliar a freqüência de uso da internet e de jogos eletrônicos, mostrou que a maioria dos participantes era do sexo masculino $(62,5 \%)$ e $75 \%$ tinham idade entre 25 e 30 anos. Medindo o nível de experiência com a internet, numa escala de 1 a 5, sendo 1 iniciante e 5 expert, 87,5\% dizem ter nível 4 ou 5, ou seja, a grande maioria dos participantes se sentem muito seguros ao utilizar a internet. Dentre os participantes, $87,5 \%$ da amostra utilizam a internet diariamente, e $25 \%$ fazem uso de jogos eletrônicos com frequência diária e outros $25 \%$ afirmam fazê-lo muitas vezes por semana; os restantes $50 \%$ raramente utilizam jogos de eletrônicos, o que foi sinalizado como frequência inferior a uma vez por semana.

Dessa forma, o grupo de foco foi conduzido em torno da discussão acerca do tema "Autoria de jogos para fins educacionais". Foi feita uma breve explanação pelo mediador relacionada ao tema e em seguida aberta a discussão questionando-se ao grupo sua opinião sobre se seria possível aprender algum conteúdo de caráter educacional através do uso de jogos. Houve consenso do grupo quanto a isso, sendo reforçado o caráter motivador e estimulante inerente aos jogos.

O participante $\mathrm{C}$ afirmou: - "Mais que possível é estimulante. Torna o aprendizado muito mais interessante" e mais adiante diz "Deixa de ser uma obrigação e passa a ser um prazer".

$\mathrm{O}$ participante A reforçou a diferença entre o aprendizado utilizando jogos e o modo convencional usando slides, quadro branco e livros.: - "A forma como aprende $o$ conteúdo faz com que ele fique mais fixo [sic] na nossa cabeça"... "Algo aprendido de forma lúdica você associa aquele bom momento, o momento divertido, não é como abrir um livro e ficar lendo, você está interagindo com a informação enquanto você está se divertindo, então aquilo entra mais fácil e se fixa mais fácil".

Ao serem questionados se eles se sentiam capazes de construir um jogo, alguns participantes, mais experientes em programação, disseram já ter tentado e desistido por demandar muito tempo de desenvolvimento. Outros disseram que sabiam que não seriam capazes devido a sua própria formação não os habilitar para desenvolvimento baseado em linguagens de programação.

Diante da discussão foram extraídos alguns requisitos necessários para serem encontrados em jogos com fins educacionais, tais como: Facilidade de Uso, Desafio, Visual atrativo, Divisão em níveis, Ranqueamento e Compartilhamento de Resultados. 


\section{Ferramenta de Autoria GReAT}

A Figura 1 mostra a arquitetura proposta para o ambiente de autoria, composta de três camadas principais: Application Layer, Content Provider e Plugin Layer.

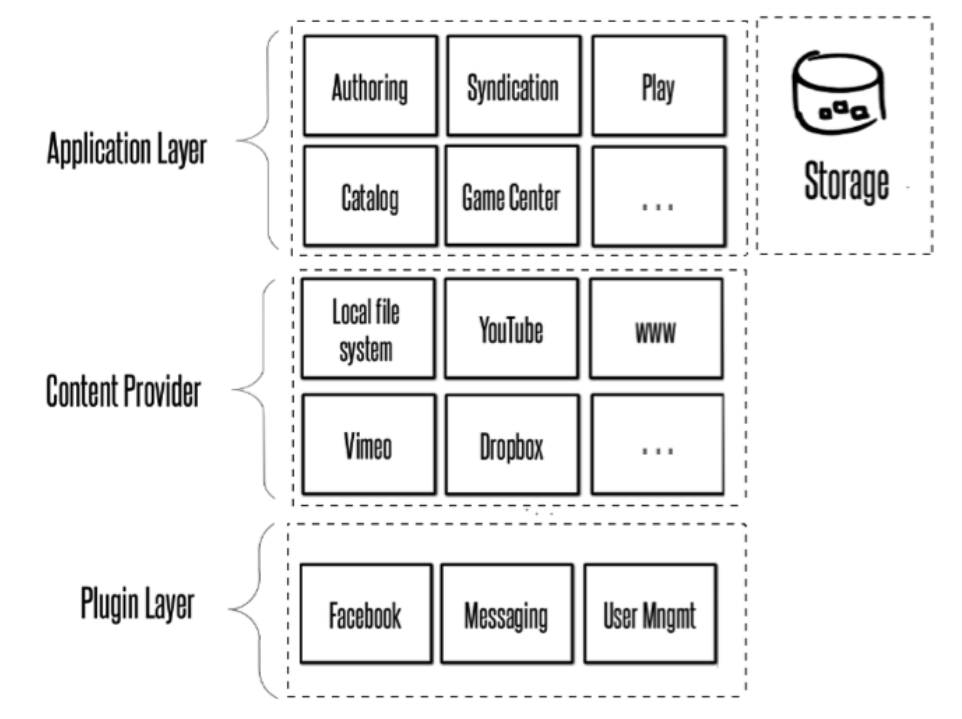

Figura 1. Arquitetura do ambiente de autoria de jogos sérios - GReAT

A camada de aplicação (Application Layer) compreende os módulos principais relacionados à ferramenta. Authoring refere-se à aplicação de autoria propriamente dita, onde, em ambiente web, o usuário poderá criar seu próprio jogo com base em templates pré-codificados; Syndication permite aos jogadores fazer assinatura de canais de jogos, como feeds RSS, podendo então ser notificados quando houver atualização de jogos de um determinado assunto ou autor. Catalog é o catálogo de jogos à disposição do usuário, indexado por nome (páginas brancas) e tipo de conteúdo (páginas amarelas). Game Center permite acompanhar o progresso de cada usuário, seu último placar, prêmios conseguidos, lista de desafios e compartilhar resultados com amigos. O módulo Play será responsável por executar o jogo propriamente dito, permitindo diferentes versões para $t a-$ blets, smartphones, desktops, além da possibilidade de jogar a partir de um navegador da web.

A camada de conteúdo (Content Provider) permite acesso a conteúdo multimídia, local ou remoto, que pode ser utilizado nos jogos, como, por exemplo, imagens, podcasts, músicas e vídeos, disponibilizados em serviços como Dropbox, Youtube, Vimeo, Google Drive e outros. Finalmente, à camada de plugins podem ser integrados componentes reutilizáveis, agregando mais valor a ferramenta como um todo, tais como integração com redes sociais, gerenciamento de usuários e contatos, envio de mensagens, download de conteúdo, entre outros.

\subsection{Diagrama Conceitual}

Na Figura 2, é apresentado um diagrama conceitual do ambiente, cujos principais pontos são descritos a seguir. 


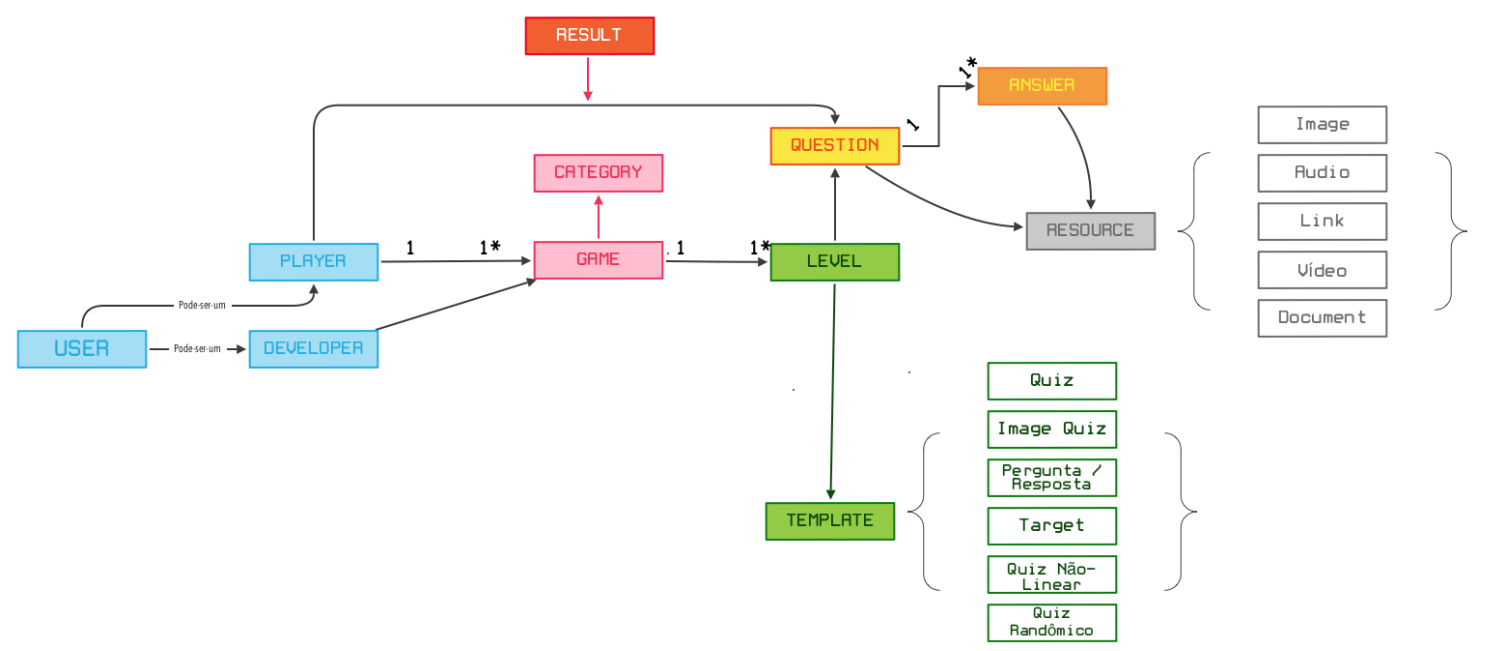

Figura 2. Diagrama Conceitual

Um usuário/ator pode assumir dois papéis na ferramenta, o primeiro como desenvolver de jogos (developer), normalmente um professor ou instrutor, que irá elaborar a dinâmica do jogo, criar os níveis e as perguntas de cada nível. E também no papel de jogador (player), quando poderá acompanhar seus resultados, assinar os canais de conteúdo de jogos, fazer o download do conteúdo para seu dispositivo (seja móvel ou desktop), e jogar.

Um jogo é dividido em níveis (levels). Cada nível apresenta um conjunto de perguntas e respostas que, a depender do template escolhido para aquele nível, assume um conjunto de características e comportamentos específicos. Os templates de níveis serão abordados a seguir.

\subsection{Templates de Níveis}

Ao cadastrar o nível, o desenvolvedor de jogos deverá selecionar um template para o mesmo. No estágio atual, foram implementados apenas templates do tipo questionário, descritos a seguir, por serem bastante comuns em cenários de ensino-aprendizagem, embora a ferramenta seja flexível para se ajustar a outros tipos de templates.

i) Quiz: Neste template de nível, é cadastrada uma pergunta e várias alternativas como resposta, onde o usuário deverá selecionar a correta;

ii) Image Quiz: Neste template, o jogador é levado a fazer uma associação da imagem que é apresentada e digitar um texto como resposta. A Figura 3 mostra um exemplo de jogo que possui níveis do tipo Image Quiz, onde o usuário deve acertar o nome do país dada sua bandeira, agrupada por continente;

iii) Pergunta / Resposta: Aqui, é cadastrada uma pergunta e sua respectiva resposta e o usuário deve escrever a resposta correta;

iv) Target: Neste template, similar ao Quiz, cada resposta é pontuada diferentemente. Sendo assim, não existe resposta errada, existe resposta mais certa ou menos certa quando comparada com as demais;

vi) Quiz Randômico: Neste template, é cadastrado para o nível um conjunto de perguntas e suas respectivas respostas (incluindo a resposta correta) e então definida 


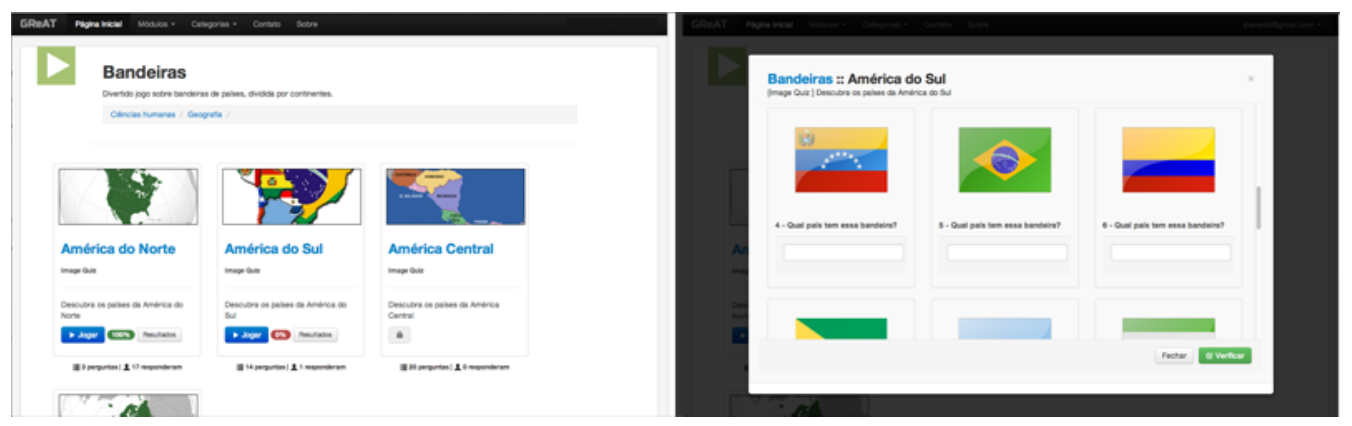

Figura 3. Exemplo de Jogo com níveis do tipo Image Quiz

uma quantidade arbitrária de perguntas que o jogador deve responder, sendo estas escolhidas de forma randômica;

vii) Quiz Não-Linear: Este template é uma variação do Quiz, onde para cada pergunta é associada a próxima questão que será exibida ao jogador, uma em caso de acerto e outra, em caso de erro. Isto implica que a questão seguinte é diferente, dependendo se o jogador acerta ou erra a pergunta.

\subsection{Ciclo de Desenvolvimento de Jogos}

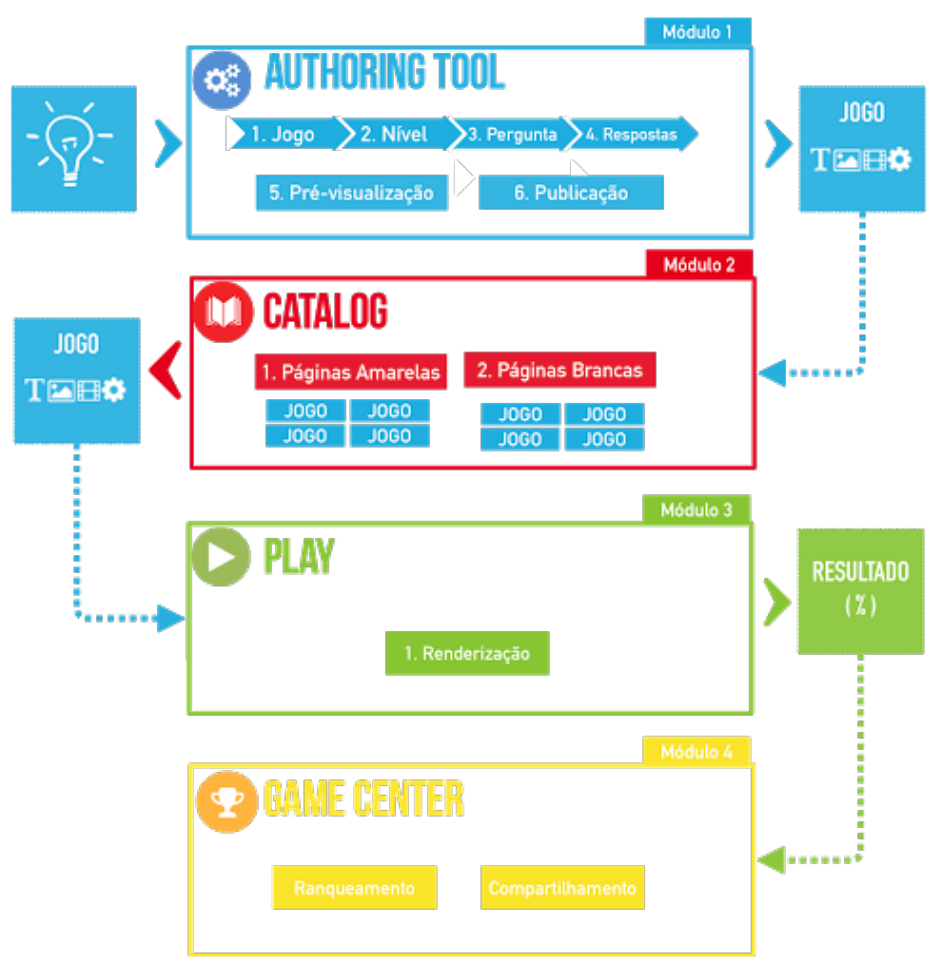

Figura 4. Processo de Desenvolvimento usando a ferramenta GReAT

O Ciclo de Desenvolvimento de Jogos usando a ferramenta GReAT vai desde a concepção do jogo até a divulgação e compartilhamento dos resultados, como mostrado na figura 4. 
No Módulo 1 (Authoring Tool), tem-se a criação do Jogo em si (Passo 1.1) através do preenchimento de um formulário onde é solicitado um nome para o jogo, uma descrição e as categorias às quais ele pertence, e uma imagem para representar o jogo. Após criar o jogo, o passo seguinte (Passo 1.2) é a criação dos níveis. Nesta etapa, serão criados quantos níveis forem necessários para completar o jogo, de acordo com o planejamento do desenvolvedor. No Passo 1.3, a elaboração das perguntas pode ser feita em paralelo com o Passo 1.4 (cadastro das respostas). Cada pergunta pode ter uma ou mais respostas certas e estas podem conter, além de texto, imagens ou vídeos. Antes da publicação do jogo, é possível fazer a pré-visualização do nível, a fim de evitar que seja publicado um nível contendo erros. A ferramenta GReAT ainda fornece, em cada uma de suas etapas, notificações automáticas que verificam possíveis erros lógicos no jogo, como jogos publicados sem níveis cadastrados, níveis sem perguntas, ou ainda perguntas sem respostas corretas cadastradas. Após o jogo ser publicado ele fará parte do catálogo de jogos da ferramenta GReAT. É a partir do catálogo que o usuário, agora no perfil de jogador irá encontrar um jogo de seu interesse para então experimentá-lo.

Segue-se, então, para a apresentação do jogo (Play), com todo seu conjunto de mídias, acrescido da configuração atribuída ao jogo recém-construído, tais como: instruções, texto e imagens do jogo, níveis, perguntas ou respostas, além de redimensionamento de imagens e vídeos. O Modulo Play ainda é responsável por calcular o resultado da partida e atribuir um percentual para o jogador no nível recém jogado. Esse percentual servirá para o cálculo total e ranqueamento dos jogadores no Módulo 4 (Game Center), onde também é possível fazer o acompanhamento dos resultados por usuário, por jogo e compartilhar os resultados em redes sociais.

\section{Avaliação de Usabilidade da Ferramenta}

Para a avaliar a Ferramenta de Autoria quanto à usabilidade, foi aplicado o formulário ISONORM 9241/110-S ([Prümper 2010]) que faz referência aos princípios de diálogo da Norma ISO 9241-110.

A ISO 9241-110:2006 (ou, sua versão em português, ISO ABNT 9241-110:2012) trata da ergonomia da interação humano-sistema e estabelece sete princípios ergonômicos de projeto formulados em termos gerais, ou seja, sem referência a situações de utilização, aplicação, ambiente ou tecnologia.

O termo "dialógo" é definido na Norma como sendo a interação entre um usuário e um sistema interativo com uma sequência de ações do usuário (entradas) e respostas do sistema (saídas). E ainda,"princípios de dialogo" como sendo um conjunto de objetivos gerais para o projeto de diálogo. Os princípios são: [1] Adequação para a tarefa , [2] Auto-descrição, [3] Conformidade com as expectativas dos usuários , [4] Adequação ao aprendizado, [5] Controlabilidade, [6] Tolerância a erro e [7] Suporte à individualização.

Segundo [Sachse and Thüring ], uma maneira eficiente para testar a usabilidade da interação humano-computador é a utilização de questionários padronizados que permitem a avaliação de vários aspectos sem a necessidade de testes em laboratório, além de que o sistema pode ser avaliado no seu contexto de trabalho natural. O formulário ISONORM 9241/110-S [Prümper 2010] aborda cada princípio sugerido na Norma ISO 9241-110, onde cada um dos princípios é considerado como uma dimensão separada e medido por três itens. A Figura 5 mostra parte do formulário, que aborda a dimensão "Adequação 
para a tarefa".

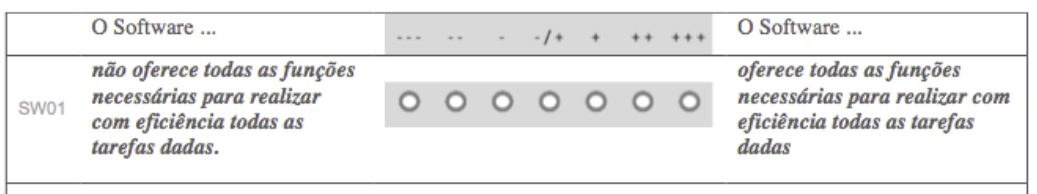

Figura 5. Exemplo de pergunta no Formulário ISONORM 9241/110-S

\subsection{Resultados Obtidos}

Para a avaliação da ferramenta, o formulário ISONORM 9241/110-S foi traduzido e aplicado a uma turma mista de alunos de Pós-Graduação em Design e em Ciência da Computação, após terem utilizado a ferramenta de autoria GReAT para a produção de jogos. Os resultados obtidos estão tabulados na Figura 6.

\begin{tabular}{|l|c|c|c|c|c|c|c|}
\hline Princípio de Diálogo & -- & -- & - & $-/+$ & + & ++ & +++ \\
\hline 1 - Adequação a tarefa & - & $4,17 \%$ & $8,33 \%$ & $12,50 \%$ & $16,67 \%$ & $50 \%$ & $8,33 \%$ \\
\hline 2 - Autodescrição & $4,55 \%$ & $4,55 \%$ & $18,18 \%$ & $31,82 \%$ & $27,27 \%$ & $13,64 \%$ & - \\
\hline 3 - Expectativa do usuário & $4,17 \%$ & $4,17 \%$ & $8,33 \%$ & $16,67 \%$ & $37,50 \%$ & $29,17 \%$ & - \\
\hline 4 - Adeq. p/ o aprendizado & - & $4,17 \%$ & - & - & $8,33 \%$ & $33,33 \%$ & $54,17 \%$ \\
\hline 5 - Controlabilidade & $4,17 \%$ & $8,33 \%$ & $12,50 \%$ & $4,17 \%$ & $12,50 \%$ & $45,83 \%$ & $12,50 \%$ \\
\hline 6 - Tolerância a erros & $12,50 \%$ & - & $16,67 \%$ & $12,50 \%$ & $16,67 \%$ & $20,83 \%$ & $20,83 \%$ \\
\hline 7 - Adeq. p/ individualização & $4,55 \%$ & $4,55 \%$ & - & $9,09 \%$ & $36,36 \%$ & $31,82 \%$ & $13,64 \%$ \\
\hline
\end{tabular}

Figura 6. Compilação dos resultados do formulário ISONORM 9241/110-S)

Pode-se observar que segundo o princípio \#1, adequação para a tarefa, a ferramenta obteve $75 \%$ de apreciação positiva (colunas,,++++++ ) por parte dos utilizadores, contra $12,5 \%$ que sinalizaram que a ferramenta não atenderia a este requisito (colunas $---,--,-)$. Os outros $12,5 \%$ dos participantes da pesquisa (coluna $-/+$ ) não sinalizaram contentamento ou descontentamento em relação a este item. Em relação ao princípio \#2, autodescrição, 27,7\% das respostas dos usuários dizem que o sistema não atende este principio. Enquanto que 31,82\% mostraram-se neutros em relação ao layout e 40,91\% sentem-se confortáveis quanto a aplicação deste princípio na ferramenta proposta. Segundo o princípio de conformidade com a expectativa do usuário, princípio \#3, 16,67\% dos usuários tiveram apreciação negativa da ferramenta, contra 66,67\% com apreciação favorável e 16,67\% neutros. O princípio que obteve melhor resultado foi o de adequação ao aprendizado, princípio \#4, onde a soma dos valores positivos atinge 95,83\%. No princípio \#5, controlabilidade, 70,83\% dos participantes mostraram-se positivos em relação à controlabilidade que a interface oferece, permitindo alternância fácil entre os menus, não sendo necessário seguir uma sequência rígida de passos para completar a tarefa. No item tolerância a erros, princípio \#6, 29,17\% dos utilizadores sinalizaram uma preocupação em relação às mensagens de erro e a ajuda efetiva que elas teriam quanto a resolução de problemas que possam ter ocorrido. Contudo, 58,33\% dos participantes consideraram adequada a aplicação deste princípio na ferramenta de autoria, uma vez que mesmo ocorrendo erros, isso não impediu que a tarefa fosse concluída, ensejando ações corretivas mínimas por parte do usuário. Por fim, quanto ao princípio \#7, que se refere a 
adequação para individualização, $81,82 \%$ dos usuários acreditam que o sistema é fácil de se adaptar a diferentes tipos de tarefas, ao estilo individual de trabalho de cada um e ainda na generalização de novas tarefas que possam ser dadas a eles.

\subsection{Discussão}

A análise dos resultados da seção anterior é claramente favorável às decisões de projeto de diálogo tomadas na concepção da ferramenta de autoria GReAT. Senão, vejamos: observa-se que um número expressivo dos usuários pesquisados, cerca de $3 / 4$ afirma inequivocamente, embora com diferentes graus de ênfase, que o ambiente GReAT é a ferramenta adequada para o fim ao qual se propõe, qual seja, o desenvolvimento de jogos com fins educacionais. Isto, em particular, nos torna confiantes das decisões de projeto tomadas e do caminho percorrido desde o levantamento de requisitos da ferramenta GReAT.

Outro resultado forte da avaliação feita está no atendimento das expectativas dos usuários por parte da GReAT, pois mais de $80 \%$ dos usuários tiveram uma apreciação positiva ou neutra quanto a esse princípio. Isto mostra que a ferramenta corresponde ao conhecimento que o usuário possui sobre a tarefa de desenvolvimento de jogos e aborda o tema usando um vocabulário convencional, reconhecido pelos usuários.

O item melhor avaliado foi a adequação da ferramenta para o aprendizado, ao qual a quase totalidade dos usuários mostrou-se favorável, o que demonstra que a GReAT requer pouco tempo para o aprendizado e facilita o reconhecimento do que fazer, ao invés da simples memorização.

Por outro lado, a maioria dos usuários não concorda que a ferramenta seja autodescritiva, o que significa que nem sempre as telas de diálogo são imediatamente compreensíveis ao usuário ou são fornecidas explicações sensíveis ao contexto realmente úteis. Isto indica que a ferramenta deve ser melhor trabalhada no sentido de melhor situar o usuário em que estágio da tarefa este se encontra e o que é esperado dele naquele ponto.

Outro ponto forte do sistema, apontado pela avaliação feita está no controle que os usuários têm sobre a tarefa que estão desempenhando, o que foi confirmado por mais de setenta por cento de respostas positivas. Dessa forma, os usuários não se sentem presos a uma sequência rígida de passos ou menus, o que certamente tem um impacto positivo na flexibilidade da ferramenta e, por conseguinte, na produtividade dos seus usuários.

Por fim, vale destacar a facilidade de adequação/individualização da ferramenta GReAT, apontada por cerca de 4/5 dos usuários pesquisados, o que implica que a GReAT adapta-se de forma descomplicada ao estilo individual de trabalho e às habilidades de cada usuário, sendo ainda facilmente generalizável no caso de novas tarefas surgirem.

\section{Conclusão}

Este artigo descreveu um ambiente de autoria para o desenvolvimento de jogos sérios, que permite a professores, instrutores e outros profissionais produzir jogos de computadores com fins educacionais, em diversos níveis de dificuldade e com propósitos diversos. A ferramenta desenvolvida, denominada GReAT é baseada na Web e por essa razão não exige nenhum tipo de configuração no ambiente computacional do usuário, estando imediatamente disponível. Com o ambiente GReAT, torna-se possível criar jogos multimídia a partir de diferentes templates pré-codificados, como Quiz, Image Quiz, 
Pergunta/Resposta, Target, Quiz Randômico e Quiz Não-Linear, além de outros que poderão ser-lhe incorporados no futuro. A GReAT possui também um Catálogo de Jogos, onde os alunos (jogadores) podem selecionar o conteúdo desejado, utilizando diferentes filtros. Fornece ainda um Game Center, por meio do qual os jogadores podem publicar o resultado dos seus jogos e comparar com os dos seus colegas, através de um sistema de ranqueamento. A ferramenta GReAT também apresenta integração com conteúdos disponíveis na nuvem, Internet e redes sociais, maximizando assim as possibilidades de criação dos jogos.

O ambiente GReAT foi validado com usuários potenciais (professores e alunos de diversas áreas) e teve sua usabilidade formalmente avaliada através da aplicação da Norma ISO 9241-110, no contexto de uma disciplina de Pós-Graduação conjunta com alunos dos Mestrados em Ciência da Computação e em Design da UFMA. A apreciação dos usuários sobre a GReAT foi bastante positiva, em especial no tocante às dimensões de adequação à tarefa, atendimento das expectativas dos usuários, adequação ao aprendizado, controlabilidade e individualização, conforme Norma acima. Nessa avaliação, foram apontadas também melhorias a serem feitas nos aspectos de autodescrição e tolerância a erros da ferramenta.

Como trabalhos futuros, destacam-se: a) desenvolvimento de uma versão da ferramenta GReAT para tablets e smartphones; b) exportação dos jogos desenvolvidos para o formato SCORM, a fim de permitir sua integração com Ambientes Virtuais de Aprendizagem; c) realização de uma Avaliação Heurística segundo os princípios de Nielsen; d) avaliação da usabilidade dos objetos (jogos) produzidos pela ferramenta; e) desenvolvimento de novos templates de jogos; e) implementação de canais de assinatura de distribuição de jogos na ferramenta, dentre outros.

\section{Referências}

Antunes, A. M. and Sabóia-Morais, S. M. T. O jogo educação e saúde: Uma proposta de mediação pedagógica no ensino de ciências (the game education and health: a proposal for pedagogical mediation in science teaching).

Barbosa, S. and da Silva, B. (2010). Interação humano-computador. Elsevier.

Battistella, P. E. and von Wangenheim, A. (2011). Avaliação de ferramentas de autoria gratuitas para produção de objetos de aprendizagem no padrão scorm. Revista Brasileira de Informática na Educação, 19(03):16.

de Lara, I. C. M. (2004). Jogando com a matemática de $5^{\mathrm{a}}$ a $8^{\mathrm{a}}$ série.

ElearningBrasil, S. (2013). Games aplicados ao e-learning. http://www . elearningbrasil.com.br/pesquisa/resultados/pesq_result_ 137. asp. [Online; accessed 11-December-2013].

Machado, L. S., Moraes, R. M., and Nunes, F. (2009). Serious games para saúde e treinamento imersivo. Abordagens Práticas de Realidade Virtual e Aumentada, 1:31-60.

Nielsen, J. (1997). The use and misuse of focus groups. IEEE Softw., 14(1):94-95.

Prümper, J. (2010). Isonorm 9241/110-s: Evaluation of software based upon international standard iso 9241, part 110. [Online; accessed 4-November-2013].

Sachse, K. and Thüring, M. A usability evaluation tool for safety-relevant systems. 